\title{
SCIENTIFIC ORGANIZING COMMITTEE
}
G.S. Bisnovatyi-Kogan
P. Bodenheimer
S. Hayakawa
R. Kippenhahn
D.Q. Lamb
B. Paczyński
M. Plavec
D.N. Schramm
D. Sugimoto (Joint Chairman)
R.J. Tayler (Joint Chairman)

LOCAL ORGANIZING COMMITTEE
S. Hayakawa
R. Hoshi
N. Itoh
T. Matsuda
T. Nakano
K. Nakazawa
H. Sato (Chairman)
D. Sugimoto 